\title{
La prevención secundaria con gemfibrozil reduce la mortalidad y el riesgo de infarto de miocardio
}

\author{
Bloomfield Rubins H., Robins S.J., Collins D. y col. N. Engl. J. Med. 1999, 341: 410-418
}

\section{Objetivo}

Determinar si el incremento del colesterol $H D L$ y la reducción de los niveles de triglicéridos mediante el tratamiento con gemfibrozil en pacientes coronarios que presentan niveles bajos de HDL y LDL, reduce la mortalidad y la incidencia de infarto agudo de miocardio.

\section{Diseño}

Estudio prospectivo, multicéntrico, randomizado, doble ciego controlado contra placebo.

\section{Lugar}

Veinte instituciones médicas pertenecientes al Departamento de la Administración de Veteranos de EE.UU

\section{Pacientes}

Se reclutaron 2531 pacientes de sexo masculino con enfermedad coronaria documentada que tenían menos de 74 años de edad, niveles de $\mathrm{HDL}<40 \mathrm{mg} / \mathrm{dl}$ y de $\mathrm{LDL}<140 \mathrm{mg} / \mathrm{dl}$. El seguimiento promedio fue 5,1 años. La edad media fue $64 \pm 7$ años, infarto previo $61 \%$ y revascularización coronaria previa $56,5 \%$.

\section{Intervención}

Los pacientes fueron asignados a recibir en forma aleatorizada gemfibrozil ( $n=1264)$ o placebo $(n=1267)$. Los pacientes asignados a tratamiento activo recibieron gemfibrozil a una dosis de $1200 \mathrm{mg} /$ día. Todos los pacientes recibieron instrucciones de cumplir dieta hipolipemiante.

\section{Medición del resultado principal}

El punto final primario del estudio fue la incidencia combinada de infarto de miocardio no fatal o muerte cardiovascular.

\section{Resultados Principales}

El cumplimiento del tratamiento asignado fue similar entre los pacientes asignados a gemfibrozil y a placebo ( $75 \%$ en ambos grupos). Las mediciones de lípidos al año de tratamiento demostraron que el nivel medio de HDL era un $6 \%$ mayor en el grupo gemfibrozil respecto al placebo (34 vs $32 \mathrm{mg} / \mathrm{dl}, \mathrm{p}<0,001$ ), el colesterol total un 4\% menor (170 vs 177 $\mathrm{mg} / \mathrm{dl}, \mathrm{p}<0,001$ ) y los triglicéridos un 31\% menores (115 vs $166 \mathrm{mg} / \mathrm{dl}$, $p<0,001)$. No existieron diferencias en el nivel medio de LDL.

Durante el seguimiento se observaron los siguientes eventos cardiovasculares mayores:

\begin{tabular}{lcclc}
\hline Evento & $\begin{array}{c}\text { Placebo } \\
(\%)\end{array}$ & $\begin{array}{c}\text { Gemfibrozil } \\
(\%)\end{array}$ & $\begin{array}{l}\text { Reducción de riesgo } \\
\text { relativo (\%) [IC95] }\end{array}$ & P \\
\hline $\begin{array}{l}\text { Infarto no fatal o } \\
\text { muerte cardiovascular }\end{array}$ & $275(21,7)$ & $219(17,3)$ & $22[7$ a 35] & 0,006 \\
\hline $\begin{array}{l}\text { Infarto no fatal, muerte } \\
\text { cardiovascular 0 a }\end{array}$ \\
\begin{tabular}{l} 
ccidente cerebrovascular \\
\hline Infarto no fatal
\end{tabular} & $258(20,4)$ & $24[11$ a 36] & $<0,001$ \\
\hline Muerte cardiovascular & $118(14,5)$ & $146(11,6)$ & $23[4$ a 38] & 0,02 \\
\hline Mortalidad total & $220(17,4)$ & $198(15,7)$ & $11[-8$ a 27] & 0,23 \\
\hline
\end{tabular}

No se observaron diferencias en la incidencia de cáncer entre los grupos gemfibrozil y placebo ni tampoco en el número de pacientes con elevaciones de CPK o enzimas hepáticas. La incidencia de enfermedad biliar fue similar en ambos grupos, pero los pacientes del grupo gemfibrozil reportaron dispepsia con mayor frecuencia.

\section{Conclusiones}

El tratamiento con gemfibrozil resultó de utilidad para reducir la incidencia de eventos cardiovasculares mayores en pacientes coronarios con niveles reducidos de HDL y LDL.

Fuente de financiamiento: Programa de estudios cooperativos del Departamento de la Administración de Veteranos de la 0ficina para la Investigación y el Desarrollo, y Parke-Davis Inc.

\section{COMENTARIO}

Un nivel de colesterol $\mathrm{HDL}$ reducido $(<40 \mathrm{mg} / \mathrm{dl}$ ) constituye un factor de riesgo mayor e independiente para el desarrollo de aterosclerosis, hecho que ha sido aceptado en los recientes consensos para la prevención de la enfermedad coronaria de la Sociedad Europea de Cardiología y para la evaluación del riesgo cardiovascular del AHA/ACC Task Force ${ }^{1-2-3}$. Los estudios recientes que han utilizado estatinas en el contexto de prevención secundaria (4S, CARE, LIPID) han demostrado en forma concluyente el beneficio del tratamiento para reducir los eventos cardiovasculares mayores ${ }^{4-5-6}$. Es por este motivo que se ha generalizado el empleo de estatinas para el tratamiento de los desórdenes lipídicos con elevación de los niveles de colesterol LDL por encima de $130 \mathrm{mg} / \mathrm{dl}$ (prevención secundaria) o de $160 \mathrm{mg} / \mathrm{dl}$ (prevención primaria). Un porcentaje importante (25 al $40 \%$ ) de pacientes dislipidémicos presenta sin embargo niveles de LDL por debajo de esas cifras asociados a HDL por debajo de $40 \mathrm{mg} / \mathrm{dl}$ y hasta la fecha ningún tratamiento había demostrado efectividad para reducir los eventos cardiovasculares mayores en los mismos. El presente trabajo constituye un aporte fundamentat en ese sentido, pues demuestra que en este grupo de pacientes un tratamiento que eleva los niveles de HDL sin afectar los niveles de LDL reduce en forma significativa la incidencia de muerte o infarto no fatal, despejando la incógnita de cómo efectuar el manejo de este grupo de pacientes. Por otra parte los resultados de este trabajo apoyan las afirmaciones de los consensos europeos y americanos de elevar a la categoría de factor de riesgo mayor a un nivel de $\mathrm{HDL}<40 \mathrm{mg} / \mathrm{dl}$.

Podemos concluir este comentario destacando la relevancia de los siguientes datos en el contexto de la prevención secundaria:

1- En pacientes con $L D L>130 \mathrm{mg} / \mathrm{dl}$ el tratamiento con estatinas es de elección para reducir la incidencia de eventos coronarios mayores (4S, CARE, LIPID) ${ }^{4-5-6}$.

2- En pacientes con LDL por debajo de $130 \mathrm{mg} / \mathrm{dl}$ las estatinas no han demostrado beneficios concluyentes, motivo por el cuál basándonos en los resultados del presente ensayo, en pacientes que además presentan $\mathrm{HDL}$ reducidas el tratamiento con gemfibrozil es efectivo para disminuir la incidencia de eventos mayores.

\section{Dr. Ernesto Ferreirós}

Médico Cardiólogo - Regional Clinical Data Manager, South LatinAmerica, Pfizer Lab.

Referencias

1. Bloomfield Rubins H., Robins S.J., Collins D. y col. Gemfibrozil for the secondary prevention of coronary heart disease in men with low levels of high density lipoprotein cholesterol. N. Engl. J. Med. $1999,341: 410-418$

2. Wood D., De Backer G., Faergeman 0., Graham I., Mancia G., Pyörälä K. Prevention of coronary heart disease in clinical practice. Recommendations of the Second Joint Task Force of European and other Societies on Coronary Prevention. Eur. Heart J. 1998, 19: 1434-1503

3. Grundy S.M., Pasternak R., Greenland P., Smith S., Fuster V. Assessment of cardiovascular risk by use of multiple-risk-factor assessment equations. A Statement for healthcare professionals from the AHA / ACC. J. Am. Coll. Cardiol. 1999, 34: 1348-1359

4. Scandinavian Simvastatin Survival Study Group. Randomised of cholesterol lowering in 4444 patients with coronary heart disease: the Scandinavian Simvastatin Survival Study (4S). Lancet 1994, 344: $1383-1389$

5. Sacks F.M., Pfeffer M.A., Moye L.A., y col. The effect of pravastatin on coronary events after miocardial infarction in patients with average cholesterol levels. N. Engl. J. Med. 1996, 335: 1001-1009 6. The Long-Term Intervention with Pravastatin in Ischemic Heart Disease (LIPID) Study Group. Prevention of cardiovascular events and death with pravastatin in patients with coronary heart disease and a broad range of initial cholesterol levels. N. Engl. J. Med. 1998, 339: 1349-1357 